\title{
Eliminationsversuch der Nase bei den Amphibienlarven.
}

\author{
Yon \\ Chikanosuke Ogawa. \\ Inatomisches Institut der Kaiserlichen Lniversität zu Kyoto.
}

Mit 1 Textfigur.

Entwicklungsmechanische Experimente kömmen an den Sinnesorganen der Amphibienlarven ohne besondere Schwierigkeit vorgenommen werden. Bisher sind aber die einzelnen Organe nicht gleich häufig experimentell studiert worden, und zwar das Sehorgan am meisten, das Gehörorgan schon viel seltener und endlich das Geruchsorgan, oder in anderen Worten die Nase, am seltensten. Da die Nase einfachere Form- und Baurerhältnisse darbietet, hat sie rielleicht nur wenig das Interesse der Forscher auf sich gezogen.

Das vorliegende Experiment bezweckt in der Hauptsache festzustellen, ob bei der Bildung der Nase und des Ductus nasolacrimalis eine etwaige Korrelation zwischen beiden Gebilden besteht, anders ausgedrückt, ob die Bildung des Ductus ron der Nase abhängig ist oder nicht.

\section{Material und Methode.}

Das Experiment erstreckte sich über 2 Jahre, und zwar wurde die Operation im ersten Jahr (1925) an der Larve der Rana nigromaculata, im zweiten Jahr (1926) an der des Bufo vulgaris japonicus vorgenommen.

Die Ranalarven sind $5 \mathrm{~mm}$ lang; das Auge ist pigmentiert und ebenso etwas das Nasengrübchen, welches ein wenig eingesunken ist. Die Bufolarven befanden sich in einem späteren Stadium und waren $2 \mathrm{~cm}$ lang. Ton Extremitäten war noch nichts zu bemerken; Nase und Auge waren schon ausgebildet. 
Zur Verfügung standen 35 Rana- und 70 Bufolarven, von denen später nur an wenigen die Operation gelang.

Die Operation wurde an den Bufolarven unter Chloroformnarkose zuerst mit der Nadel unter dem Binokularmikroskop ausgeführt. Ich zerstörte die Nasenhöhlenwandung mit der Nadel und manipulierte damn so, dass das Lumen durch die gelockerte Zellmasse verstopft wurde. Im weiteren Vorlauf wurde aber die nach der Operation verschlossene Nasenhöhle wieder durchgängig. Die Operation war also misslungen. Ich benutzte alsclann eine glüh(nde Nadel, um die Zerstörung noch eingreifender durchzuführen. Doch auch diesmal war der Erfolg ungenügend. Ich will daher über den Versuch mit den Ranalarven nichts weiter berichten.

In nächsten Jahre machte ich bei der Opcration an den Bufolarven Gebrauch von einem durch clektrischen Strom glühend gemachten sehr feinen Nichromdraht. Diesmal erzielte ich mehr oder weniger das gewünschte Resultat. Obwohl auch hier in der grösseren Mehrzahl der Experimente die Nasenhöhle wieder hergestellt wurde, kam es doch bei einer kleinen Anzahl von Fällen auf der operierten Seite gerade bis zur Metamorphose zum Verschlusse der Nasenhöhle. Fixiert wurde \& Wochen bis 80 Tage nach der Operation in Sublinatpikrinsäure. Die Tiere besassen vollständig ausgebildete Extremitäten und machten die Metamorphose durch.

Icli habe im ganzen 11 Exemplare mikroskopiert, welche äusserlich kein Nasenloch aufwiesen. Zur Kontrolle mikroskopierte ich einige operierte, doch äusserlich mit dem Nasenloch versehene Exemplare. Um einerseits über den normalen Verlauf der Bildung des Tränennasengangs bei Bufo Auskunft zu erhalten und andererseits den mikroskopischen Befund der Nase und deren Umgebung un die Zeit der Operation kemmen zu lernen, wurden ausserdem alle Serien von normalen Bufolarven Schritt für Schritt unter dem Mikroskop studiert.

Zunächst möchte ich angeben, was ich bei Born über die normale Entwicklung des Ductus nasolacrimalis kemmen gelernt habe.

Nach Born bildet sich der Tränenkanal der Amphibien durch Finwachsung und Abschnürung eines Epithelstreifens von der Nase bis zum Auge hin, der dann ein Lumen bekommt und sich mit der Nasenhöhle in Verbindung setzt. ..... Bei Rana beginnt noch vor der Einsenkung der Epithelzapfen zur Bildung der Hautdrüsen die Einwachsung des Tränenkanals...... Zuerst bemerkt man an der Apertura narium externa, durch einen etwas erhöhten Rand von ihr getrennt, eine Einwachsung des Epithels in die Cutis, die man von da noch eine strecke 
weit nach hinten verfolgen kann; dam flacht sie sich ab und wird allmählich ummerklich. Ein direkter Übergang des eingewachsenon Epithelstreifens in das Epithel des Nasenlochs ist auf den Schnitten kaum nachweisbar, lässt sich aber mit genügende! Sicherheit aus don späteren Bildern folgern. In der Regel fällt die erste Bildung des Tränenkanals bei Rana mit dem Auftreten der ersten Verknorpelung in der Ungebung der Riechsäcke zusammen, doch kommen erhebliche individuelle Schwankungen nach oben und unten vor. Ein weiterer Fortschritt bestcht darin, dass der eingewachsene Epithelstreif sich nach dem Auge hin verlängert, sich gleichzeitig mit seinem vorderen Ende von der Epidermis abschnürt und in die Tiefe wandert. ...... Bald schnürt sich die Einwachsung ihrer ganzen Länge nach ron der Epidermis ab, und as wird in ihr ein Lumen deutlich.

Aus dieser Beschreibung erhellt ohn' weiteres, dass der Ductus nasolacrimalis sich vielmehr sekundär in Verbindung mit Nase und Auge setzt.

Nach meinen Präparaten im Stadium der Operation bildet sich ausser dem Planum internasale noch kein Epithelstreifen aus der Haut in der Umrandung des äusseren Nasenlochs und keine Knorpelkapsel in der Umgebung der Nase. Man bemerkt hier und da in der Epidermis Leydig'sche Epithelzellen, aber keine Epithelzapfen als Anlage der Hautdrüsen. Selbst an den Präparaten in noch späteren Stadien ist noch nicht der Epithelstreifen des Ductus bemerkbar.

Bei meiner Operation ist also der Ductus nasolacrimalis sicher noch nicht in Bildung begriffen.

Nach dieser Vorbmerkung gehe ich zu meinen eigentlichen Untersuchungen über.

\section{Beschreibung der Experimente.}

A. 7 Wochen post operationem: Nase und Ductus nasolacr. nicht vorhanden.

Auf der normalen Seite ist die Nasenhöhle mit einer Knorpelkaysel umgeben und hat sich auch das Jacobsonsche Organ gebildet. Auf ler Seite der Operation fehlt eine eigentliche Knorpelkapsel ausser derverdickten medialen, welche sich ron der normalen Seito kontinuierlich nach der Seite der Operation erstreckt. Die Nase fehlt spurlos, ebenso das Jacobsonsche Organ.

Die Haut auf der Seite der Operation sieht ganz normal aus und beherbergt Hautḍrüsen, welche nach Zahl und Bau völlig normal sind. Die Gaumendrüse ist auf beiden Seiten normal ausgebildet. 
Auf der normalen Seite ist ein vollständig ausgebildeter Ductus nasolacr. zu sehen, während auf der Seite der Operation auf der ganzen Strecke zwischen Nase und Auge kein Ductus gefunden wird.

B. 2 Monate p. op.

Auf der Scite der. Operation bemerkt man eine Nasenhöhle, welche in grossen und ganzen normale Form besitzt. Jedoch fehlt ihr die äussere Öffnung, und die immere ist so verengt, dass die Öffnung nur auf einem einzigen Schnitt sichtbar ist. Die Höhle enthält eine durch die Fixationsflüssigkeit erzeugte geromnene Masse. Die Zellschicht ist dünner als gewöhnlich.

Das Bild lehrt, dass die einmal durch Zexstörung geschlossene Nasenhöhle später wahrscheinlich wieder durchgängig wurde. Die Erweiterung der Höhle ist vielleicht auf die durch Verschluss hervorgerufene Retention und den dadurch verursachten erhöhten Innendruck zurückzuführen.

Auf der Soite der Oporation findet sich der Ductus nasolacrimalis ganz in normaler Lage vor, nur dass das untere Ende ganz nahe bei der Nascnwandung blind endigt, ohne in die Höhle zu münden.

C. 2 Monate p. op.

Auf der Seite der Operation stellt die Nascnhöhle ein allseitig geschlossines kleines Bläschen dar. Die Epithelschicht der Wandung ist lümn, und das Lumen enthält eine geronnene Masse. Die umgebencle Knorpelkapsel ist verdickt. Das Jacobsonsche Organ ist nur rudimentär ausgebildet. Iuf der Seite der Operation entspringt der Ductus nasolacrimalis an normaler Stelle und endigt nach kurzem Verlauf ohne sich irgend zu öffnen, blind unter der Epidermis.

D. 80 Tage p. op.

Auf der Seite der Operation stellt die Nase eine allseitig geschlossene Höhle dar, wolche nur mit dem Jacobsonschen Organ in Kommunikation stcht. Die Nasenhöhle ist von einer Knorpelkapsel umgeben.

Der Ductus nasolacrimalis entspringt an normaler Stelle, durchbohrt, ohne die Nasenregion zu erreichen, die Epidermis und öffnet sich nach aussen mit ganz enger Öffnung.

E. 80 Tage p. op.

Die Nasenhöhle ist an der Seite der Operation völlig geschlossen und enthält eine seröse, gerommene Masse. Die Wandschicht ist dünn. Krin Jacobsonsches Organ. Der Ductus nasolacrimalis befindet sich in normaler Lage und endigt blind, ohne in die Nasenhöhle zu münden, ventralwärts von der Nasengegend unter der Epidermis.

F. 55 Tage p. op. 
Die Nase ist auf der operierten Seite nicht vorhanden. Dic Knorpelmasse füllt kontinuierlich von der gesunden Seite her den betreffenden Raum aus. Der Ductus nasolacrimalis endigt unter der Epidermis blind.

G. 53 Tage p. op.

Die Nase fehlt auf der operierten Seite vollständig. Auch vom Ductus nasolacrimalis ist nichts zu sehen.

H. 2 Monate p. op.

Die Nasenhöhle auf der operierten Seite ist der Gestalt nach fast normal ausgebildet, nur dass sich nach aussen und inmen keine Kommunikation feststellen lässt. Das Lumen enthält eine gerommene Masse. Die Wanddicke ist normal. Der Ductus nasolacrimalis ist vorhanden. Er öffnet sich nicht nur unten mit enger Öffnung in die äussere Haut, sondern auch in die Nasenhöhle.

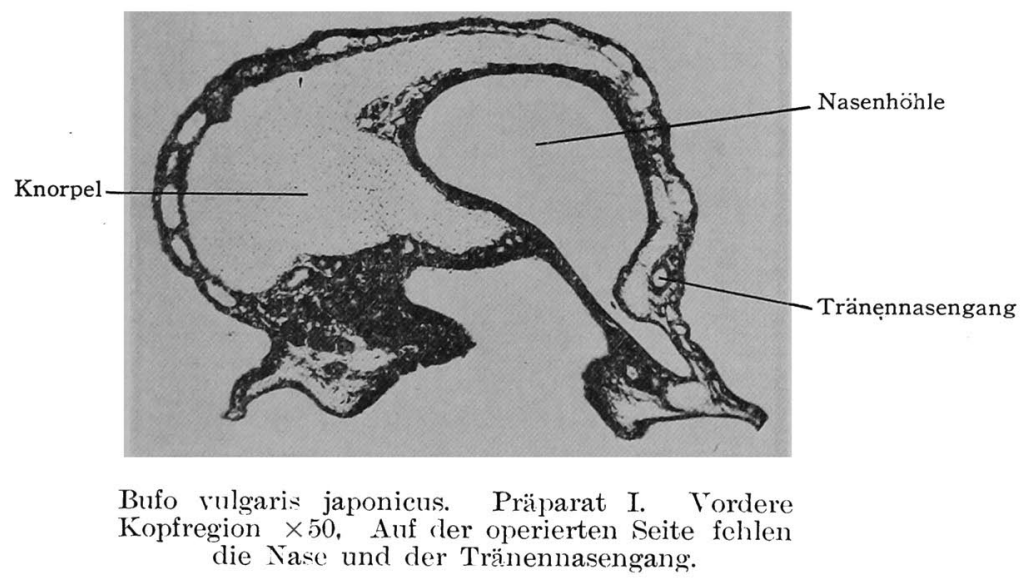

I. 8 Wochen p. op.

Auf der operierten Seite fehlt die Nase gänzlich. An der vorderen Kopfregion nimmt dic Nase auf der anderen Seite den wesentlichen Teil der Region ein, während nach hinten der Bezirk der eliminierten Nase durch eine verdickte, mit der linken Nasenkapsel in kontinuierlicher Verbindung stehenden Knorpelmasse ausgefüllt ist. Nach dem Ductus nasolacrimalis sucht man vergebens.

J. 8 Wochen p. op.

Die Nase fehlt auf der operierten Seite. Doch ist der Ductus nasolacrimalis vorhanden und öffnet sich unten durch die Haut nach aussen, nur dass das untere Ende ganz nahe am Munddach liegt.

K. 8 Wochen p. op. 
Die Nasenhöhle ist partiell gebildet. $\mathrm{D} \times \mathrm{r}$ Ductus nasolacrimalis endigt blind unter der Epidermis, ohne sich in die Nasenhöhle zu öffinen.

\section{Besprechung der Befunde.}

Nach den Resultaten der Experimente von Steinitz und Osaki an Amphibienlaren bildete sich der Ductus nasolacrimalis aus, nachden der Augapfel im Frühstalium enukleiert worden war. Da sich im I'rlauf der normalen Entwicklung der Ductus zuerst in der Nasengegend zu bilden beginnt und sich damn nach dem Auge hin erstreckt, um in dessen Umgebung sein Ende zu finden, war dieses Resultat mehr oder weniger zu erwarten. Die Bildung des Ductus nasolacrimalis ist also vom Augapfel unabhängig, oder in anteren Worten: Der Augapfel bestimmt oder leitet die Bildung des Ductus in keiner Weise.

M(in Experiment spricht vielmehr für die Abhängigkeit des Ductus ron der Nase.

Unter 11 mehr oder weniger gelungenen Fällen findet man in 3 Fällon keinen Ductus nasolacrimalis und in 5 keine Nasc. Diese 3 Fälle, wo der Ductus nasolacrimalis fehlt, finden sich unter diesen 5 Fällen olıne Nase, was sicher auf einen Zusammenhang zwischen Nase und Ductus hinweist, da man hier nicht gut von einem Zufall sprechen kamn. Ich möchte auf Grund nocines Resultats behaupten, dass sich der Ductus nur gebildet hat, weil eben schon eine Nase zur Ausbildung gelangt war, oder anders ausgedrückt, dass die Bildung des Ductus von der Nase abhängt. Da die erste Anlage des Ductus regionär nur nahe an der Nase, nicht aber direkt aus der Nase vor sich geht, dürfte diese Abhängigkeit zwischen Ductus und Nase nicht bloss mechanischer Natur sein. Man darf hierbei viclleicht an eine Taxis denken, deren Wesen uns jedoch, wis bei vielen anderen Fïllen, noch unklar ist.

Bei 2 Fällen unter 5 findet sich Bildung des Ductus nasolacrimalis, ohne dass eine Nase vorhanden wäre. Danach scheint auf den ersten Blick keine Abhängigkeit zwischen beiden Gebilden zu bestehen. Bei genauer Überlegung fand ich aber für diese Fälle folgende Erklärung, die nicht ins Reich der Unmöglichkeit gehört. Obwohl im Operationsstadium noch keine Anlage des Ductus nasolacrimalis sichtbar ist, kamn doch die Epidermis des betreffenden Bezirks ron. der Nase her schon dalıin beeinflusst sein, dass sich später ein Gang bildet.

Nach Born unterliegt die Bildung des Tränennasengangs grossen individuellen Schwankungen. Wenn dem wirklich so ist, kamn auch die Determination dieses Gangs individuell in verschiedenen Stadien 
erfolgen. Danach könnte bxi den 3 Fällen mit fehlendem Ductus die Nase schon vernichtet gewesen scin, als der betreffende Lpidermisbezirk noch nicht von der Nase her determiniert war, während bei den 2 Fällen, wo der Ductus nasolacrimalis trotz des Fehlens der Nase zur Ausbildung gelangte, die Nase erst vernichtet wurde, nachdem die betreffende Epidermis schon unter dem Einfluss der Nase zur Bildung des Gangs determiniert worden war. So hatte sich also trotz Abwesenheit der Nase ein Tränennasengang gebildet. Da es im allgemeinen genug Fälle gibt, bei denen sich eine Anlage vor ihrem Sichtbarwerd(n determiniert, so hat meine Anmahme viel Wahrscheinlichkeit für sich. Um diese Wahrscheinlichkeit aber zur Gewissheit zu erheben, ist es nötig, die Operation an Larven in noch jüngeren Starlien auszuführen. Nur ist in diescn Stadien das Gelingen der Operation sehr zweifelhaft.

Dagegen, dass der. Ductus nasolacrimalis von der Nase abhängig gebildet wird, lässt sich noch einiges einwenden. Bei der Zerstörung der Nasenhöhle durch den glühenden Draht wird die Haut in der Umgebung des Nasenlochs natürlich mehr oder weniger mitverbrannt. Danach könnte das Fehlen des Tränemmasengangs darauf zurückzuführen sein, dass die Anlage desselben durch die Hitze vernichtet und demgemäss die nachherige Entwicklung des Gangs unmöglich wird. Dem ist aber gegenüberzuhalten, dass in anderen Fällen trotz mancher Abnormitäten, ja trotz vollständigen Verschlusses der Ö̈ffuung, stets cin Tränennasengang sichtbar ist. Es ist danach nicht anzunehmen, dass die Mitverbrennung der umgebenden Haut vernichtend wirkt. So darf man wohl behaupten, dass der Ductus von der Nase abhängig gebildet wird.

Wenn man das Abhängigkeitverhältnis der Nase und des Gangs analytisch betrachtet, sind zwei Möglichkeiten denkbar. Entweder wirkt die Nase determinierend auf die ganze Strecke des Ductus oder auf die erste Bildungsstätte allein ein, da sich die letztere ganz nahe bei der Nase findet. Ob das der. Fall ist, ist ohne weiere Experimente nicht gut zu entscheiden.

Unter zahlreichen Experimenten kam es nur an einer relativ kleinen Anzahl von Fällen (11) zu einem äusserlich sichtbaren Verschluss. Unter diesen wenigen Fällen fand man wieder nur 5 Fälle, bei denen die Nasenhöhle vollständig vermisst wurde. In anderen Fällen waren die Höhlen immer crhalten, auch wonn die äussere oder die immere Öffnung geschlossen war. Ich habe auch einige äusserlich mit Nasenlöchern versehene Individuen mikroskopiert und komnte dabei ausnahmslos eine ganz normale Ausbildung der Nasenhöhlo konstatieren. Auch 
an ander'n gleichen Exemplaren wird die Nase vielleicht normal ausgebildet gewesen sein.

An diesen Präparaten selbst war die Operation so gewaltsam ausgeführt worden, dass dic Nase dabei vollständig mit zerstörter Gewebsmasse ausgefüllt war. Und doch waren die Nasen später an zahlreichen. Fällen normal oder fast normal ausgebildet, was für die grosse Regulationskraft der Amphibiennasen spricht. Da nun die Präparate der Zwischenstadien nicht zur Hand sind, kann ich zurzeit nicht mit Sicherheit aussagen, ob einmal desquamierte Epithelzellen sich wieder zusammenfügen und eine neue Nasenwandung bilden, oder ob die von der Desquamation verschont gebliebenen Epithelzellen sich regenerieren und die Bekleidung bilden, oder ob endlich dic Nasenepithelzellen einmal mitsamt der darunterliegenden zerstörten Mesenchymmasse zugrunde gehen und ron neuem die Mundschleimhaut- oder Hauptepithelzellen eingewachsen sind und dann die Nasenhöhle decken.

Hand in Hand mit der Anomalie der Nase stösst man häufig auf Anomalie des Tränennasengangs, indem dieser Gang unten entweder blind oder offen in der Haut endet.

Zuweilen liegt der Gang subepithelial und bietet ein Bild dar, nach dem ar sich gut in die Haut öffnen könnte und doch blind endigt. Dieses Verhalten zeigt, dass keine starke Tendenz für den Gang vorhanden ist, sich irgendwohin zu öffinen.

In allen Fällen war die Schichtung der Wandung des Gangs im grossen und ganzen normal. Nan traf niemals cinen Gang mit einfachem Epithel an.

\section{Schluss.}

Bein Bufo ist die Bildung des Tränenkanals von der Nase abhängig.

\section{Literatur.}

1. Born, (i. Die Nasenhöhlen und der Tränennasengang der Amphibien. Morph. Jahrb., Bd. 2, 1876.

2. Ekman. G. Über die Regeneration der Nascnanlage bei Rana fusca. Soc. Sci. Fenn. Comment. Biol., Bd. 1, 1923. (Nach Anatom. Ber., Bd. 1).

3. Hinsberg, V. Die Entwicklung der Nasenhöhle bei Amphibien. Arch. f. mikr. Anat., Bd. 58, 1901.

4. Osaki, D. Embryonale Korrelation zwischen Auge und anderen Organen (Japanisch). Nihon Ganka Gakkai Zasshi, Bd. 30, 1925.5.

5. Steinitz, E. Über den Einfluss der Elimination der embryonalen Augenblaserk auf die Entwicklung des (iesamtorganismus beim Frosche, Arch. f. Entw:mech., Bd. $\bullet 0,1906$. 